\title{
Charismatisch beten. Perspektiven und Erfahrungen ${ }^{1}$
}

Willibald Sandler

Als Theologe und Leiter eines kleinen Gebetshauses habe ich mit charismatischem Beten sowohl aus eigener Erfahrung als auch in der theologischen Reflexion zu tun. Diesen zwei miteinander wechselwirkenden Zugängen entsprechen die beiden Teile dieses Beitrags. Die theoretisch-theologische Beschreibung und Verortung von charismatischem Beten wird im zweiten Teil auf eigene Erfahrungen mit einigen neueren Entwicklungen charismatischer Spiritualität hin durchsichtig gemacht.

\section{Was heißt charismatisch Beten?}

Charismatisch Beten ist ein vom Heiligen Geist geleitetes Beten. ${ }^{2}$ Als solches ist es nicht nur betende Bewegung auf Gott hin, sondern von Gott her auf ihn hin. Als Betender gebe ich Gott Raum in meinem Leben, sodass Er in mir und mit mir wirken kann. Dieses Wirken geschieht in einer die menschliche Freiheit und menschliche Vermögen nicht außer Kraft setzenden, sondern freisetzenden Weise durch den Heiligen Geist.

\subsection{Charismatisch beten und der Kairos einer unverfügbar einbrechenden Gotteserfahrung}

Dieser Ansatz muss präzisiert werden: Dass der Betende Gott Raum in sich gibt - indem er sich in der Ganzheit seiner Personalität dazu bestimmt, sich von Gott bestimmen zu lassen - vermag er nicht mit den Kräften seiner (gefallenen) Natur. Normalerweise haben

\footnotetext{
1 Eine ausführlichere, umfassender dokumentierte Fassung dieses Aufsatzes findet sich online unter http://theol.uibk.ac.at/itl/1060.html.

2 "Auch für Paulus ist jedes echte Gebet pneumatisches, inspiriertes Gebet. Der Heilige Geist treibt Menschen zum Gebet (Röm 8,14f) und macht sie ihrer Gotteskindschaft gewiß (Röm 8,15f; Gal 4,6f). Darum können sie zu Gott »durch Christus" und "im Geist " beten. Charismatiker haben diese Aussagen neu zu Gehör gebracht und auf die Wichtigkeit der pneumatischen Dimension des Gebets hingewiesen« (P. Zimmerling, Die charismatischen Bewegungen. Theologie - Spiritualität Anstöße zum Gespräch, Göttingen $\left.{ }^{22} 2002,220\right)$.
} 
wir uns nicht so weit in der Hand, dass wir uns selber wirklich schenken könnten. ${ }^{3}$ Für den Grundvollzug von Freiheit, in dem ein Mensch sich vor Gott auf Gott hin entscheidet, ist ihm zwar ein fundamentales Vermögen in die Wiege gelegt, welches aber faktisch meist durch vielfältige Bedingtheiten zugedeckt ist. Es ist ein Kairos, das heißt ein unverfügbares Gnadenereignis, das seine souverän von Gott festgelegte Zeit hat, in der einem Menschen Gott neu und tiefer aufleuchtet, sodass er im Licht dieser Selbstoffenbarung sich selbst neu und tiefer in seinem Verhältnis zu Gott bestimmen kann und es damit auch muss: in einem Ja oder einem Nein.

Wie in den biblisch bezeugten Begegnungen von Menschen mit Jesus kann ein solcher Kairos ungefragt und sogar ungewollt über Menschen hereinbrechen. Dadurch werden sie für eine begrenzte Zeit von der erfahrenen Präsenz Gottes mitgerissen. ${ }^{4}$ Hierin können ekstatische Momente in der Gotteserfahrung, die ja für charismatische und pentekostale Spiritualitäten zentral sind, einen authentischen Ursprung haben. Die zeitweilige Wucht oder "Gewalt« der erscheinenden Herrlichkeit Gottes (biblisch: Kabod) schließt nicht aus, dass Gottes Wirken in souverän von Ihm verfügten Begegnungen die menschliche Freiheit respektiert, sie also nicht zerstört, sondern vielmehr freisetzt. Die eigentliche Freiheit einer Selbstbestimmung des Menschen in Bezug auf Gott setzt erstens voraus, dass Gott dem Menschen trotz aller erbsündig-konkupiszenten Gottesverfinsterung überhaupt erst erscheint und so zum Gegenstand seines Wahlvermögens wird. Das geschieht in der Weise, dass der Mensch angesichts des sich offenbarenden Gottes sich selbst zum Gegenstand seiner Wahl wird, was nicht ohne Durchbruchserfahrung möglich ist, die unter Umständen als unerwartet, unerwünscht, erschreckend

\footnotetext{
3 Selbsthingabe - an Gott oder auch an einen Mitmenschen - ist dem Menschen grundsätzlich nur vermittelt möglich. Ich gebe mich selbst, indem ich etwas gebe, was meine Selbsthingabe realsymbolisch vergegenwärtigt. Schon die Gegebenheit dafür geeigneter Gegenstände in einer geeigneten Situation stellt einen Kairos dar - einen unverfügbaren Gnadenmoment.

4 Eine solche Gotteserfahrung kann ausdrücklich als solche wahrgenommen werden oder auch implizit sein, wie etwa in Jakobs Traum: „Der Herr ist an diesem Ort, und ich wusste es nicht" (Gen 28,16). Sie kann spektakulär oder auch sanft verlaufen, "als ein sanftes, leises Säuseln" (1 Kön 19,12). Auch im letzteren Fall ist etwas Mitreißendes dabei, dem man sich nicht ohne weiteres entziehen kann. Es kann über längere Zeit wirksam sein oder nur einen flüchtigen Augenblick anhalten (vgl. dazu unten die Anm. zu Lk 4,16-30). Ich vertrete die These, dass ein solches Durchbruchsmoment der Gotteserfahrung, wenn auch nur flüchtig, so doch in jedem Fall gegeben ist. Dieses Moment kann leicht übersehen und vernachlässigt werden, wenn man dem Prinzip folgt, dass Gott gewaltlos ist und Freiheit achtet, ohne die spezifische Zeitlichkeit von Ereignissen der Gottesbegegnung zu berücksichtigen.
} 
oder gewaltsam erfahren wird. ${ }^{5}$ Zweitens ist für eine freigesetzte Freiheit des Menschen Gott gegenüber erfordert, dass der Mensch angesichts des sich offenbarenden Gottes dennoch die Möglichkeit hat, sich gegen Gott zu entscheiden. Das erste wird durch die - unter Umständen als gewaltsam erlebte - Durchbruchserfahrung eines Kairos der Gottesbegegnung ermöglicht, das zweite durch einen gewissen Rückzug Gottes im Gefolge dieser Erfahrung, durch ein Verblassen des Kairos, wodurch nun auch Vorbehalte und Optionen wieder hochkommen können, die ein Ja zu Gott behindern. ${ }^{6}$ In dieser Situation im Gefolge eines Kairos kann und muss eine - erneute, vertiefte - Entscheidung für oder gegen Gott gefällt werden. Das ist die eigentliche Zeit des Glaubens: als Festhalten an einerVerheißung, die mit einer Gotteserfahrung verbunden ist, welche nun nicht mehr präsent ist, aber erinnert werden kann. In diesem Sinn gilt hier: "Selig sind, die nicht sehen, aber doch glauben" (Joh 20,29), und zwar nicht im Gegensatz zu Gotteserfahrungen, sondern - in der Weise einer glaubend vollzogenen Erinnerung - auf ihrer Grundlage. Glaubend wird das, was Gott im Kairos seiner geschenkten Begegnung offenbart hat, für das eigene Leben ergriffen: Das solcherart Ergriffene ist Gott selbst, aber gewöhnlich vermittelt durch einen bestimmten Auftrag oder auch durch eine konkrete Zusage neuer Lebensmöglichkeiten. Zum Beispiel in der Erfahrung von Heilung oder Besserung von Lebensbedingungen: "Dein Glaube hat dir geholfen «?

In der eigenen Lebensgeschichte gibt es wenige große, lebensverändernde und zahllose kleine, geradezu übersehbare Kairoi von Gotteserfahrung. Angesichts dieser Vielfalt leben wir ${ }^{8}$ immer auch

\footnotetext{
5 Nach dem Lukasevangelium erschrickt selbst Maria, als sich ihr Gott vermittels eines Engels offenbart, trotz ihrer Sündenlosigkeit.

${ }^{6}$ Ein solches Ablehnung ermöglichendes Verblassen des Kairos kann viel später oder auch beinahe unmittelbar erfolgen. Für Letzteres gibt die Predigt Jesu in seiner Heimatstadt Nazaret ein biblisches Beispiel (nach Lk 4,16-30), wo die Synagogenbesucher nach anfänglicher Zustimmung beinah sofort in eine - kollektiv verstärkte - Skepsis verfallen. Vgl. dazu W. Sandler, Kairos und Parusie. Kairos als Ereignis des in Christus angekommenen und angenommenen Gottes, in: ZKTh 136 (2014), 10-31, hier: 11-15; etwas ausfuihrlicher im Internet: http://theol.uibk.ac.at/itl/1018. html\#ch3

7 Mt 9,22; Mk 5,34;10,52; Lk 7,50; 8,48; 17,19; 18,42.

8 Dieses "Wir» kann auf beinahe alle Menschen generalisiert werden, wenn man berücksichtigt, dass Glaubenserfahrungen auch implizit bleiben können (nach Karl Rahner etwa im Vollzug von Nächstenliebe, Zukunftshoffnung und Annahme des Todes; vgl. seine "suchende Christologie« in: Ders., Grundkurs des Glaubens, Freiburg 1976, 288-291) und dass sie - gerade in solch impliziter Form - nicht nur spektakulär-lebensverändernd, sondern so unauffällig und alltäglich sein können, dass
} 
von Gotteserfahrungen her, die unserem Glauben Halt geben: einem Glauben, der durch das Zeugnis anderer - vor allem im biblischen und kirchlich verkündeten Gotteswort - geformt wird, aber durch eigene Glaubenserfahrungen erst einen existentiellen Anhalt gewinnt. Die im freien oder (biblisch, liturgisch) geformten Gebet dankbar vollzogene Erinnerung an große und kleine Taborerfahrungen, in denen Gott in unserem Leben aufgeleuchtet ist, kann die Sehnsucht anfachen, Seine Gegenwart neu und tiefer zu erfahren.

Damit ist ein Sinnzusammenhang von Gotteserfahrung, Glaubensentscheidung und Gebet eröffnet, innerhalb dessen sich charismatisches Beten angemessen beschreiben lässt, und zwar in einer Weise, die (1.2) gewisse Schlagseiten charismatischer Spiritualität von ihrer Mitte her zu kritisieren erlaubt und die (1.3) nicht exklusiv ist, sondern eng verbunden mit einer christlichen Grunderfahrung, welche für jeden Christen relevant ist.

\subsection{Kritik und Unterscheidung von der "kairologischen « Mitte der charismatischen Spiritualität her}

Als typisch für charismatisches Beten gilt, dass es expressiv und emotional ist: mit erhobenen Händen und ekstatischem Sprachengebet. Die emotionale Erhebung, die durch ihren gemeinschaftlich vollzogenen, unbefangenen Ausdruck in körperlichen Bewegungen, Singen und "feurigem" Bezeugen leicht auf andere übergreifen kann, deckt einerseits Defizite von traditionellen Frömmigkeitsformen ab, bringt aber auch Gefahren von Suggestion, Manipulation und Erlebnissucht mit sich. Wo derartiges auftritt, behindert es eine authentische Gotteserfahrung im Sinn eines unverfügbaren Kairos, der den Menschen in eine Situation der Entscheidung stellt. Ein Kairos der Gotteserfahrung lässt sich erbitten, aber nicht "machen «. Machen lassen sich stets nur Ersatzbildungen, Simulationen, Begleiterschei-

wir viele Male täglich an Gott vorbeilaufen. Bildet man eine Folge von spektakulären zu immer kleineren, unauffälligeren Formen der Gotteserfahrung, so ergibt sich ein Kontinuum zwischen kairologisch verfassten Ereignissen, in denen Gott ins Leben einbricht und einer beständigen Gegenwart Gottes. Analog ergibt sich ein Kontinuum zwischen kairologisch verfassten, intermittierenden Gnadenmomenten und einer mit der menschlichen Verfasstheit als Geschöpf von Gott gegebenen "Grundgnade» (Karl Rahner spricht hier von einem übernatürlichen Existential), die als Fähigkeit, Gottes Selbstmitteilung zu rezipieren, gemäß Gottes allgemeinem Heilswillen für jeden Menschen jederzeit gegeben ist und dennoch selber als gnadenhaft bestimmt werden kann bzw. muss. 
nungen einer echten Gotteserfahrung, die dieser im Wege stehen, wenn sie anstelle von Gott selbst angestrebt werden.

Die Ausrichtung auf Gott selbst kommt in der charismatischen Erneuerung vor allem durch den Lobpreis in Gebet und Gesang zum Ausdruck. Hier geht es ganz um die Verherrlichung Gottes, die in selbstvergessener Freude vollzogen wird, ohne dabei auf Gottes Gaben zu schauen, welche im Bitt- und Dankgebet stärker im Blick sind. Auch das charismatische Bittgebet ist primär Bitte um das Kommen von Gott selber, näherhin um den unverfügbaren Kairos einer neuen und vertieften Gotteserfahrung, einer Erhebung von innen, die dem Heiligen Geist zugeschrieben wird.

Solche Bitte um das Kommen des Heiligen Geistes ist auch im Wissen möglich, dass Gott immer schon »da« ist. Die Mangelsituation, um deren Überwindung man Gott anfleht, besteht nicht in Gottes Abwesenheit, sondern darin, dass wir erst ansatzweise "da", "gegenwärtig" sind und so dem bereits gegenwärtigen Gott weder begegnen noch uns ihm schenken können. So bitten Christen mit dem Ruf »Komm, Heiliger Geist" um den Kairos eines erfahrbaren Einbrechens Gottes in das eigene Leben, in einer Begegnung, die die eigene Präsenz in Erkennen und Fühlen gerade nicht auslöscht, sondern intensiviert gegenwärtig setzt.

Dem entspricht der ausgeprägte Erfahrungs- und Erlebnisbezug von charismatisch orientierten Christen. Er wird theologisch erst problematisch, wenn die Zielrichtung eines Kairos übersehen wird: ein vertieftes Ja zu Gott als Antwort auf die erfahrene vertiefte Selbstoffenbarung Gottes. Dieses Ja ist in der charismatischen Erneuerung zu einer festen Form geworden im Gebet der Lebensübergabe, die nicht nur einmalig als Antwort auf die charismatische Anfangserfahrung erfolgt, sondern immer wieder wiederholt wird, etwa durch das Singen entsprechender Lieder. Erst dort, wo die Ernsthaftigkeit der auf die Gotteserfahrung antwortenden Selbsthingabe ausfällt, droht eine charismatische Erlebnissucht. Der Zusammenhang von Gabe (eines gesteigerten Erlebens), Aufgabe (einem Sendungsruf, zu dem die erhaltene Gabe Ausstattung ist) und Hingabe (in gehorsamer Erfuillung des Rufs, als Konkretisierung der Lebensübergabe) ist auch maßgeblich für die - in der charismatischen Erneuerung naturgemäß zentralen - Charismen. Diese sind nicht Selbstzweck oder Belohnung, sondern Ausrüstung für die Christus Nachfolgenden. 


\subsection{Charismatisches Beten im Zusammenhang eines spiritualitätsübergreifenden, vom Heiligen Geist geleiteten Betens}

Wird charismatisches Beten wie hier als vom Heiligen Geist geleitetes Beten verstanden, dann ist klar, dass es ein solches nicht nur im Zusammenhang eines Kairos der Gottesbegegnung - als besonderem Wirken und Rufen Gottes - gibt, sondern auch im Zusammenhang von Gottes allgemeinem, nicht an existentielle Ausnahmesituationen und ekstatisches Erleben gebundenen Heilswirken. Dazu gehört vor allem das sakramentale Beten, zum Beispiel in der eucharistischen Epiklese. Hier ist Gottes heilswirkende Präsenz der Gemeinde bzw. dem Christen mit jener zweifelsfreien Gewissheit zugesagt, die Jesus nach biblischem Zeugnis jenen Menschen versprochen hat, die in seinem Namen bitten.

Diese verschiedenen Formen eines geistgeführten Betens schließen sich nicht aus, sondern ergänzen sich gegenseitig. Liturgisch geformtes Beten, das dem weiten Bogen der Heilsgeschichte und den Zyklen des Kirchenjahrs folgt, eröffnet einen Leben, Welt und Geschichte übergreifenden Rahmen heilvoller göttlicher Präsenz, in den die besonderen Gotteserfahrungen, die im kairologisch orientierten charismatischen Beten akzentuiert werden, einfließen können. Ohne diesen Rahmen droht charismatisches Beten aktualistisch und haltlos zu werden: als ob Gott nur dort gegenwärtig wäre, wo Seine Wirkungen ekstatisch verspürt werden. ${ }^{9}$ Umgekehrt kann bei einem Beten, das sich auf Sakramente und liturgische Formen beschränkt, eine konkrete, persönliche Wegerfahrung des eigenen Lebens mit Gott unterbelichtet bleiben. ${ }^{10}$

\footnotetext{
9 "Die Ablehnung der traditionellen Liturgie bedingt das Fehlen des Kirchenjahrs und der Klage im charismatischen Gottesdienst. Beides gehört zu den besonders auffalligen Merkmalen der charismatischen Gottesdienstkultur und führt zu bedenklichen Wirklichkeitsverlusten, so daß der charismatische Gottesdienst nun seinerseits von Monotonie bedroht ist. Indem er ausschließlich auf das spontane Wirken des Geistes in der Gegenwart ausgerichtet ist, fällt der Horizont für sein Wirken in der Vergangenheit weg, der durch die Feste des Kirchenjahres vergegenwärtigt wird « (Zimmerling, Die charismatischen Bewegungen, 240).

${ }^{10}$ Sakrament und "Salbung" - letztere gemäß charismatischer Diktion im Sinn einer kraftvoll spürbaren Gottesgegenwart (biblisch mit Bezug auf die Vollmacht/exousia des Wirkens Jesu, sowie auf die Rede von Salbung in 1 Joh 2,20.27) - sind zwei Formen von Gottesgegenwart, die sich nicht ausschließen, die aber auch nicht einfach zusammenfallen. Dass Gottes sakramentale Gegenwart die höchste Form Seiner Präsenz ist, obwohl Seine Kraft und Wirkung in anderen Situationen (z.B. gemeinsamen charismatischen Gebets) oft viel stärker erlebt wird, ist für viele Christen irritierend.
} 
Dass Gott für jeden Menschen, nicht nur für Priester, Pfarrerinnen und Ordensleute, einen besonderen Ruf - konkretisiert in einer Vielzahl kleinerer und größerer Rufe - hat, ist eine Schlüsseleinsicht innerhalb der charismatischen Erneuerung. Wer Gottes großen und kleinen Rufen für sein Leben folgt, gelangt in Bereiche, wo er nicht nur Werke für Gott, sondern Werke von Gott vollbringt ${ }^{11}$ und dafür Gottes Kraft und Seine Gnadengaben - Charismen - empfängt. Charismatisches Beten ist ein Beten, das sich in solcher Nachfolge bewegt oder den Ruf in solche Nachfolge erbittet. Als solches ist es geleitet und begleitet von Charismen: unter anderem des Sprachengebets, seiner Auslegung, sowie der Prophetie, die Gottes besonderen Ruf - in der Zusage von Gabe und Aufgabe - für bestimmte Menschen in bestimmten Situationen präzisiert, sowie der Unterscheidung der Geister, mit der solche Zusagen auch kritisch geprüft werden.

\section{Eigene Erfahrungen mit charismatischem Beten}

Vor mehr als dreißig Jahren kam ich als junger, ziemlich verklemmter Physikstudent in einer Lebensumbruchzeit mit der charismatischen Erneuerung in Berührung und dadurch wieder zu einem lebendigen christlichen Glauben. Faszination und Irritation an charismatischer Literatur motivierte mich damals zu einem Studienwechsel in die Theologie, um für mich und andere "diesen Dingen auf den Grund zu gehen«. Ich hatte mich dann bald von

\footnotetext{
Allerdings kommt es nicht nur vor, dass charismatische Christen der Liturgie und den Sakramenten entfremdet werden, sondern es kommt auch in offenbar zunehmendem Maße vor, dass durch die charismatischen Grunderfahrungen Christen eine tiefere Liebe zu den Sakramenten gewinnen.Vgl. dazu: International Catholic Charismatic Renewal Services [ICCRS] - Theologischer Ausschuss, Taufe im Heiligen Geist, hg. v. Vorstand der Charismatischen Erneuerung in der Katholischen Kirche Deutschland, Maihingen 2013, 20f. Charismatisch geprägte Eucharistiefeiern waren seit Beginn des innerkonfessionellen charismatischen Aufbruchs zentral, und in mehreren charismatischen Erneuerungsbewegungen sind Eucharistie, Sakramente und sakramentale Spiritualität zuinnerst mit charismatischen Formen verbunden. Theologisch sind für das Verhältnis von Sakrament und Salbung noch Klärungen nötig, auch als ökumenische Frage im Verhältnis zu Freikirchen.

${ }^{11}$ Nach dem Johannesevangelium sagt Jesus, dass er nur die Werke vollbringen kann, die er seinen himmlischen Vater tun sieht (Joh 5,19). Dem entspricht es, wenn der Epheserbrief sagt, dass wir »in Christus Jesus geschaffen zu guten Werken [sind], die Gott vorher bereitet hat, damit wir in ihnen wandeln sollen« (Eph 2,10 ELB).
} 
der charismatischen Spiritualität entfernt, bis es mich zwanzig Jahre später - als doktorierter Universitätsassistent für Theologie - wieder in die charismatische Erneuerung zu ziehen begann. Einige Jahre lang besuchte ich charismatische Gebetskreise und Veranstaltungen als einfacher Teilnehmer, ohne als Vortragender oder Leiter eigene theologische Kompetenz einzubringen. Vor sieben Jahren gründete ich einen Gebetsraum mit zweimal täglichen Gebetszeiten, zuerst in der eigenen Wohnung, dann seit zwei Jahren ein kleines Gebetshaus in Innsbruck. ${ }^{12}$

Weil ich selber an den meisten Gebetszeiten teilnehme, bedeutet das, dass ich seit sieben Jahren viele Stunden wöchentlich in einer charismatisch und kontemplativ ausgerichteten Weise bete. Diese Praxis hat die innere Ausrichtung meines Lebens tief verändert und auch Auswirkungen auf meine Weise, Theologie zu treiben. Umgekehrt hat die fortlaufende theologische Reflexion zu einer eigenständigen Ausrichtung charismatischer Spiritualität beigetragen. ${ }^{13}$

\section{$2.1 »$ Vom Nicht-Tun zum Mit-Tun«}

Die charismatische Expressivität mit den erhobenen Händen weckte in mir anfangs Widerstand. Je mehr die Menschen um mich herum vor allem bei Großveranstaltungen - die Hände und ihr Gemüt erhoben, desto stiller wurde ich. ${ }^{14}$ Ein Vortragender empfahl Neulingen, einfach so zu tun, als ob man »Charismatiker« wäre. Man würde dann von selber hineinwachsen, bis die erhobenen Hände zum authentischen Selbstausdruck würden. Ich probierte das aus und es "funktionierte" auch. Später hatte ich aber den Eindruck, auf einen anderen Weg geführt zu sein. Wenn in Gemeinschaft charismatische Lieder gesungen wurden, begann ich einfache, kleine Bewegungen

\footnotetext{
${ }^{12}$ Vgl. die Homepage http://dieweide.org mit Texten zur dort praktizierten kontemplativ, charismatisch und sozial orientierten Spiritualität.

13 Diese fließt auch in Vorträge und Seminare ein, die ich seit einigen Jahren für die Charismatische Erneuerung Österreichs halte.

${ }^{14}$ Das entspricht der Feststellung von Peter Zimmerling: „Nicht jedes Gemeindeglied ist in allen drei Bereichen von Geist, Seele und Leib gleich stark ansprechbar. Gerade eine ganzheitlich verstandene Lobpreiskultur muß dem einzelnen die ihm entsprechende Auswahl der Ausdrucksmöglichkeiten überlassen. Nur so entspricht sie der Vielfalt des Geisteswirkens. Mit der Neuentdeckung der emotionalen und körperlichen Dimensionen von Lobpreis und Anbetung ist häufig eine Unterschätzung des Verstandes verbunden. Stark verstandesmäßig orientierte Menschen werden dadurch leicht abgestoßen oder fühlen sich unter Druck gebracht, im Gottesdienst emotional und körperlich zu agieren" (Zimmerling, Die charismatischen Bewegungen, 223).
} 
auszuführen, wenn sie einer inneren Bewegung entsprachen. Das führte dazu, dass ich etwa mitten in einer Liedstrophe ein wenig meinen Arm zur Seite streckte, während meine Hände beim Refrain, wo die meisten die Arme hoben, unten blieben. Anfangs musste ich mich überwinden, solche unangepassten Bewegungen zuzulassen, dann fiel es - in einem Umfeld, das körperliche Expressivität zuließ - zunehmend leichter und ich entwickelte eine Freude und Freiheit mich zu bewegen, die weit über das hinausging, was sich früher mit dem angeleiteten Tun-als-ob ergeben hatte. Eine willkommene Nebenwirkung war, dass ich mich mehr und mehr frei fühlte von jeder Art von Gruppendruck. ${ }^{15}$

Später entwickelte sich aus diesen Erfahrungen das Prinzip:»Vom Nicht-Tun zum Mit-Tun." Danach steht am Anfang der jeweiligen Gebetszeit ein mehr kontemplatives Ankommen in der Gegenwart Gottes, gemäß der Einsicht: Gott ist immer schon da, aber ich bin noch nicht da. ${ }^{16}$ Er ist da und wartet auf mich. Er will sich mir geben und Er will mich mir geben. Wie Maria, die Schwester Martas, sitze ich zu den Füßen Jesu, bereit, alles von Ihm aufzunehmen. Was ich mitbringe an Stimmungen oder auch Ablenkungen (Gedanken, Sorgen, Pläne), halte ich Ihm hin, mit dem Anfangsgebet "Alles in deine Hände«. Erst wenn es zu einer inneren Bewegung kommt, in der ich die Präsenz Gottes spüre, drücke ich diese Bewegung aus, indem ich ein Gebet spreche (kurz, frei), ein Lied anstimme, eine Bibelstelle lese oder meine innere Bewegung in eine körperliche Bewegung übersetze. Dieses Mit-Tun ist Zustimmung, Akklamation zu dem, was ich als Gnadenzuwendung Gottes erfahre. Die Evangelien bezeugen, wie wichtig ein solches Zustimmen ist, damit Gott den nächsten Schritt führen und Verheißungen umsetzen kann.

Die Bewegung "Vom Nicht-Tun zum Mit-Tun« kann auch im gemeinschaftlichen Gebet vollzogen werden. Wenn ich in einen Raum komme, wo charismatisch gebetet wird, dann muss ich nicht sofort mitmachen! Selbst wenn es sehr laut zugeht, was bei freikirchlichcharismatisch ausgerichteten Großveranstaltungen oft der Fall ist,

\footnotetext{
${ }^{15}$ Die expressive Spontaneität in der charismatischen Erneuerung ist dennoch nicht frei von der Gefahr, konformistisch zu erstarren. "Auch dieVielfalt verstandesmäßiger, emotionaler und körperlicher Ausdrucksmöglichkeiten bietet keine Garantie für die Geistbewegtheit von Lobpreis und Anbetung. Die charismatische Lobpreiszeit kann genauso wie das traditionelle Gotteslob einer geisttötenden Ritualisierung anheimfallen" (Zimmerling, Die charismatischen Bewegungen, 223).

${ }^{16}$ Eine solche natürliche Verbindung von charismatischem und kontemplativem Beten findet sich auch bei Johannes Hartl, der ein charismatisch ausgerichtetes Gebetshaus in Augsburg gegründet hat.Vgl. ders., In meinem Herzen Feuer. Meine aufregende Reise ins Gebet, Witten 2014, 58f.
} 
kann ich mich in einen Raum innerer Ruhe versenken und still für mich beten: "Jesus, ich will nur dich. "Erst wenn mich eine Bewegung aus meinem Umfeld in meiner betenden Ausrichtung auf Gott hin unterstützt, beginne ich mitzumachen: nicht notwendig konform und so viel oder wenig, wie es der inneren Bewegung entspricht.

Auch als Leiter einer Gebetszeit bin ich in unserem Gebetshaus frei. Ich muss nicht ein bestimmtes Programm vorbereiten und "machen". Ich tauche ein in die Gegenwart des großen, unfassbaren Gottes, und erst wenn eine innere Bewegung da ist, mache ich mit. Gebete, Liedvorschläge und körperliche Bewegung können unterdessen von anderen Anwesenden kommen. Wenn sich aber auch bei ihnen nichts zeigt, oder wenn die anwesenden Besucher zu scheu sind, eigene Impulse einzubringen, können damit auch längere Zeiten der Stille entstehen. Meist aber ist es umgekehrt: Bereits beim Betreten des Gebetsraums spüre ich, wie Gott mich in seine Gegenwart zieht und ich beginne mit Gebet und Liedern.

Damit ist der Ablauf einer Gebetszeit nicht vorhersehbar. Fix sind nur der Anfang - mit einem kurzen, freien Gebet, das Dank für die Gegenwart Gottes und die Gemeinschaft vor Gott ausdrückt, sowie die Bereitschaft zur Selbsthingabe: »Alles in deine Hände« - und das Ende mit einem gemeinsamen Vaterunser.

Diese Form eines gemeinsamen charismatischen Betens ist individualistisch in dem Sinn, dass es ganz die persönliche Ausrichtung auf Gott betont - zu Füßen Jesu, wie Maria, die Schwester Martas ohne dabei besondere Rücksicht auf die (vermuteten) Bedürfnisse der anderen zu nehmen. Solches Beten ist aber zugleich offen auf Gemeinschaft aus der Erfahrung und dem Vertrauen, dass man dann, wenn man die Begegnung mit Gott realisiert, von Ihm in einer besseren, auch sensibleren Weise für die Situation und die Bedürfnisse der anderen Menschen geöffnet wird. Die anfängliche Nichtbeachtung von fremden Besuchern, die in die Gebetszeiten dazu stoßen, wird von diesen gewöhnlich nicht als achtlos, sondern als entlastend wahrgenommen. Sie kommen sich nicht vereinnahmt vor und sind frei, dasselbe zu tun, was sie bei den anderen, gerade auch den Leitenden sehen: sich mit der Situation, in der man ist, primär nicht auf Mitmenschen, sondern auf Gott auszurichten. Von Anfang an steht Gott im Mittelpunkt, nicht die Gemeinschaft und nicht der (bedürftige) Andere. Die Ausdrucksformen in Gesang, Gebet, Bibellesung, Bibeldeutung und Bewegung, die sich aus einer hörenden, empfangenden Grundhaltung ergeben, werden dann oft auch von anderen Teilnehmern als stimmig und gemeinschaftsoffen wahrge- 
nommen. Dies wird auch durch die Möglichkeit eines "Murmelbetens" unterstützt:Wenn Teilnehmer eine Bewegung in freiem Gebet artikulieren wollen und es sich dabei um Inhalte handelt, die nicht für alle relevant sind, sondern ihr persönliches Verhältnis zu Gott betreffen, dann beten sie nur halblaut, sodass von den anderen nicht der Inhalt, sondern nur das Murmelgeräusch verstanden wird. Dies lädt dazu ein, auch selber mit eigenen persönlichen Gebetsinhalten in das Murmeln einzustimmen. Damit wird ein sehr persönliches Beten möglich - durch das Verbalisieren mit einem ausgeprägteren "Mit-Tun« und einer ausgeprägteren Körperlichkeit, als es durch bloßes stilles Gebet möglich wäre - ohne dass es indiskret würde. Und das laute Beten bleibt dem Eindruck vorbehalten, dass die Inhalte auch für alle sind.

Entstanden ist dieses im Ansatz individualistische, in Gemeinschaft ganz auf Gott zentrierte Beten durch den Anfangseindruck, aus dem das Gebetszentrum entstanden ist: Betet, und lasst dabei die Türen offen.

Manches an dieser Gebetsform ist für die Besucher anspruchsvoller und herausfordernder, als es sonst bei Gebetstreffen in der charismatischen Erneuerung üblich ist. Wenn wie üblich eine ununterbrochene Folge von mitreißenden Liedern vorbereitet wird, die eine Band - unter Umständen minutiös geplant - ohne Unterbrechungen abspielt, dann kann ein mitreißender "Flow» entstehen, der das Erleben von Gott und Gemeinschaft unterstützt. Ich erinnere mich an den "Rat" eines Gebetskreisleiters: Wenn du erschöpft in eine Gebetszeit kommst, dann sing einige Lobpreislieder, auch wenn dir überhaupt nicht danach ist. So lange, bis du zu Gott "durchbrichst«. Dieser Rat ähnelt dem oben angesprochenen Vorschlag des Seminarleiters: So lange tun als ob, bis man hineinfindet. Die Gefahren dabei sind Aktivismus und fehlende Authentizität. Die eigene Befindlichkeit - unter Umständen von Erschöpfung, Trauer, Bitterkeit - wird missachtet, anstelle dass man sie (in einer Selbstannahme von Gott her) annimmt und transformieren lässt.

Wer solchen Prinzipien als Leiter folgt, kann leicht andere manipulieren und sich selbst aktivistisch überfordern. Wenn man damit erfolgreich ist, werden gruppendynamische Wirkungen in Gang gesetzt, die man dann allzu leicht unterschiedslos mit einem Wirken des Heiligen Geistes gleichsetzt. ${ }^{17}$ Wenn es bei den Menschen hingegen trocken bleibt und wenn die Gemeinschaft sich anscheinend nicht bewegt, dann muss man gemäß diesem Prinzip eben noch

${ }^{17}$ Zur Kritik einer fälschlich beanspruchten Unmittelbarkeit der Gotteserfahrung vgl. Zimmerling, Die charismatischen Bewegungen, 116f, $220 \mathrm{f}$. 
mehr Anstrengung hineinstecken, bis der "Flow" wieder hergestellt ist. Mögliche Nebenwirkungen sind dann geistliche Verkrampfung, Wirklichkeitsverlust und Burnout.

Auch bei uns gibt es oft mitreißenden Lobpreis - als ein unverfügbares Geschenk, das wir miteinander von Gott empfangen -, aber viele Gebetszeiten in unserem Gebetshaus sind "für charismatische Verhältnisse" vergleichsweise "trocken". Das führte auch dazu, dass Besucher, die ein Gefühl mitreißender "Salbung" erwarteten, bald wieder wegblieben. Andererseits kommen vor allem erschöpfte Menschen gerne, weil hier eine Atmosphäre ist, bei der man wirklich vor Gott zur Ruhe kommen kann. Und obwohl der verhältnismäßig große Anteil an geschwächten, auch psychisch belasteten Menschen eine Herausforderung ist, gilt auch für mich als Leiter: Gewöhnlich gehe ich gestärkter aus einer Gebetszeit heraus, als ich hineingegangen bin.Wäre es anders, dann ließe sich dieser Dienst nebenberuflich nicht bewältigen, so aber erfahre ich ihn als Stärkung und als »leichte Last« (vgl. Mt 11,28-30).

Eine Erfahrung, die sich aus solchem Beten ergibt, ist: Charismatische und kontemplative Spiritualität müssen sich nicht ausschließen. Im Gegenteil. Wenn charismatisches Beten ein Beten ist, dass sich vom Heiligen Geist leiten lässt, dann sind Achtsamkeit und Präsenz in einer hörenden, rezeptiven Haltung eine unverzichtbare Grundlage.

\subsection{Toronto-Erfahrung: Vom „Umfallen im Heiligen Geist» zum »Soaking}

Zur Vorgeschichte unseres Gebetshauses gehört eine Begegnung mit einem oft als extrem eingeschätzten pfingstlich-charismatischen Zentrum in Kanada. Ein biographisches Buch einer charismatischfreikirchlichen Missionarin hatte mich tief berührt. ${ }^{18} \mathrm{Um}$ von ihr zu lernen, besuchte ich 2007 eine Konferenz einer freikirchlichneocharismatischen Gemeinde in Toronto, wo sie als Gastrednerin eingeladen war. ${ }^{19} 1994$ war von dort eine Welle von extremeren charismatischen Manifestationen wie unkontrolliertes Zittern und ein "Lachen im Heiligen Geist" - oft "Toronto-Segen" genannt ausgegangen, die charismatische Kreise weltweit beeinflusst und auch

${ }^{18}$ H. u. R. Baker, Es gibt immer genug! Rolland und Heidi Bakers Dienst unter den Armen, Lüdenscheid 2003.

19 Toronto Airport Christian Fellowship (TACF), seit 2010 unter dem Namen »Catch the Fire Toronto«. Im Internet: http://en.wikipedia.org/wiki/Catch_the_Fire_Toronto, sowie die offizielle Homepage http://www.ctftoronto.com 
polarisiert hatte. Das schon länger verbreitete "Ruhen im Geist« - in einer mehr spektakulären Form, die in der englischen Formulierung "slain by the spirit" deutlicher zum Ausdruck kommt - war eines der auffälligsten dort praktizierten charismatischen Phänomene. Mit einer bemerkenswerten Geschwindigkeit gehen Leiter durch die Gänge und lassen Hunderte von dort stehenden Besuchern durch eine flüchtige Berührung auf die Stirn nach hinten kippen, wo sie von professionellen »Catchern« aufgefangen werden.

Das war auch schon 1994 so, als von dieser Pfingstkirche der sogenannte Toronto-Segen die charismatisch-pfingstlerischen Kreise weltweit durchwehte und auch polarisierte. Dem oft spektakulären Umfallen folgte eine Erfahrung von gottgeschenkter Entspannung und Frieden beim Liegen am Boden. Mit der Zeit begannen einige Gemeindeleiter zu begreifen, dass nicht das Umfallen, sondern das Ausruhen in Gottes Gegenwart die eigentliche Gnade war, und dass diese durch eine Sucht nach Spektakulärem oft vernachlässigt wurde. Nicht selten standen Menschen schnell wieder auf, um die umwerfende "Power of the Holy Ghost" erneut zu erleben, anstatt die ruhige, heilsame Gnade von Gottes liebender Gegenwart auszuhalten.

Aus dieser Einsicht entstand die so genannte Soaking-Bewegung ${ }^{20}$ : Man ließ das spektakuläre Umfallen einfach weg und legte sich schlicht selber hin. Eine ruhige, meist instrumentale Musik ("SoakingMusik«) von christlichen Musikern unterstützte die Atmosphäre des Friedens. Außer dem Einschalten des CD-Spielers besteht der Dienst von Soaking-Leitern lediglich darin, von einer Person zur anderen zu gehen und in einer unaufdringlichen Weise segnend die Hand auf die Schulter zu legen.

\subsection{Beten und dabei die Tür offen lassen}

Trotz mancher Irritationen hatte der Besuch der Konferenz »Soaking in God's Glory « auf mich und meine Begleiterin eine anhaltende Gnadenwirkung, die sich erst nach der Rückkehr zu entfalten begann. In uns entfaltete sich eine große, beständige Freude daran, im Gebet zu bleiben. Ein Jahr später entstand daraus der Impuls, jeden Abend und später auch jeden Morgen, "zu beten und dabei die Tür offen zu lassen«.

Das bedeutete, für jeden Besucher offen zu sein, ohne aber für den Gebetsraum zu werben oder die Gäste aktiv in eine Gemeinschaft $\mathrm{zu}$ integrieren. Eine der ersten unserer regelmäßigen Besucherinnen

\footnotetext{
${ }^{20}$ Von "soaking $=$ sich durchdringen, durchweichen lassen $(\mathrm{sc}$. durch den Heiligen Geist).Vgl. im Internet: http://en.wikipedia.org/wiki/Soaking_music
} 
war eine Lehrerin aus freikirchlichem Umfeld. Als sie das erste Mal von einer anderen Besucherin mitgenommen wurde, hatte ich den Eindruck, ich sollte nur die Augen schließen und überhaupt nichts sagen oder tun, nicht einmal eine Musik einschalten. Einzig ein prasselnder Gewitterregen war durch das geöffnete Fenster zu hören. Nach einer halben Stunde spürte ich, dass ich nun die Augen aufmachen sollte. Ich sah die beiden Besucherinnen - die einzigen bei dieser Gebetszeit Anwesenden - in tiefer Entspannung am Boden liegen. Was ich nicht gewusst hatte war, dass diese Lehrerin, die in einer freikirchlichen Gemeinde sehr aktiv gewesen war, an einem Burnout litt. In den folgenden Jahren kam sie fast täglich, ohne irgendetwas selber zu tun. Sie lag einfach nur da und empfing Stärkung.

In dieser Zeit hatten wir auch unsere ersten Kontakte mit einem Suchtkranken. Mit einer akuten Kokainsucht bat er uns, an einem Einkehrwoche unseres Gebetszentrums teilnehmen zu dürfen, in »kaltem Entzug«. Nach anfänglichem Zögern stimmten wir zu und boten ihm dort an, für ihn um die Gabe des Sprachengebets zu beten - als eine Form eines einfachen, unmittelbaren Betens, das in der großen Bedrängnis des Entzugs helfen konnte. Er empfing diese Gabe, und da er kaum ein Gebet auswendig konnte, wurde sie für ihn eine große Hilfe, überhaupt ins Gebet zu kommen. In dieser Woche begann er aus eigener Entscheidung, zu fasten und die Bibel zu lesen. Nach ungewöhnlich geringen Entzugsschmerzen kehrte er aus dieser Woche gestärkt und ermutigt zurück. ${ }^{21}$

Eine der größten Herausforderungen und zugleich reichsten Erfahrungen war der Kontakt mit einer extrem verschmutzten, inkontinenten Obdachlosen. Mehreren von uns wurde eine innige Begegnung mit dieser normalerweise unzugänglichen Frau geschenkt. Wir konnten sie, wunderbar befreit vom Ekel vor dem Gestank, herzlich umarmen und so die Schönheit eines Menschen dort erfahren, wo wir sie nie vermutet hätten. Ihr Besuch zu manchen Gebetszeiten in unserer Wohnung und später im Gebetshaus war für alle eine große Herausforderung, auch für sie selbst, da wir von ihr verlangten, dass sie sich zuvor duscht. Voll Freude und mit dröhnender Stimme sang sie in den Gebetszeiten Lieder mit. Wir sahen sie lachen und vor innerer Bewegung weinen. Einmal trafen wir sie an einem öffentlichen Platz, wo sie anstatt zu schweigen oder zu schimpfen lauthals christliche Lieder sang. Wir beteten viel für sie und auch mit ihr.Von ihrer Alkoholabhängigkeit und ihrer Fixierung auf den

${ }^{21}$ Er wurde schnell wieder rückfällig, kam aber regelmäßig zu den Gebetszeiten. Über ein halbes Jahr wurde er dann völlig frei von Drogen, bis er wieder einen schweren Rückfall hatte. 
Schmutz konnten wir sie dennoch nicht befreien. Eines Tages war sie verschwunden. Wie viel wir ihr geben konnten, wissen wir nicht. Aber wir wissen, dass wir durch die Begegnung mit ihr beschenkt wurden, auch wenn die Strapazen uns manchmal an die Grenzen unserer Kräfte brachten.

\subsection{Gebetshaus-Bewegung}

Der Schritt von einem Gebetskreis zu einem täglich geöffneten Gebetsraum oder Gebetshaus bringt nicht nur quantitative Veränderungen. In einem Gebetskreis öffnet man sein Herz für eine intime Begegnung mit Gott in der Gegenwart einer einigermaßen vertrauten, konstanten Gruppe. Bei täglichen öffentlichen Gebetszeiten wechseln die Teilnehmer in einem weit höheren Maße. So lernt man viel eher, auch in der Gegenwart von Fremden in freier Weise seinen Glauben zu artikulieren. Wenn man das regelmäßig praktiziert, verändert das auch die Wahrnehmung von Öffentlichkeit im Alltag. Es gibt eine tief eingewurzelte »Seitenblickmentalität «, ${ }^{22}$ mit der wir unbewusst die Erwartungen und Normen der Gruppe registrieren, in der wir uns gerade befinden. Für heikle Situationen ergibt das eine gesteigerte Wachsamkeit undVorsicht - eine Form von Lampenfieber. Zumindest in unserer europäischen Kultur, für die Glaube weithin Privatsache ist, behindert uns diese Seitenblickmentalität, unseren Glauben unverkrampft und natürlich inmitten einer säkularen Öffentlichkeit zu artikulieren. Ein Kreuzzeichen, Bekenntnis oder Gebet, das für uns in vertrauter Umgebung vielleicht selbstverständlich ist, unterbleibt oder droht, in eine verkrampfte Zurschaustellung abzurutschen. Durch das tägliche Beten in wechselnden Umgebungen im Gebetshaus erfuhr ich, wie diese Beschränkungen zurückgingen und eine ungewohnte Freiheit und Natürlichkeit in der Ausrichtung auf Gott entstand. Nicht überall, aber in immer mehr Situationen verlor eine zuweilen lähmende Präsenz von Öffentlichkeit an Intensität. Zugleich damit stieg die Freiheit, auf einzelne Menschen zuzugehen oder auch öffentlich zu intervenieren. So kann ich diese eigenartige Erfahrung von reduziert wahrgenommenem Öffentlichkeitsdruck in Verbindung bringen mit der biblischen Geistwirkung der parresia, eines Freimuts, der dazu befähigt, ein authentisches Glaubenszeugnis auch in Öffentlichkeit zu geben, ${ }^{23}$ in einer Liebe zugleich zu Gott und zu den adressierten Menschen.

\footnotetext{
${ }^{22}$ Vgl. dazu W. Sandler, Die gesprengten Fesseln des Todes. Wie wir durch das Kreuz erlöst sind. Kevelaer 2011, 40-47. Im Internet: http://theol.uibk.ac.at/itl/900.html ${ }^{23}$ Vgl. Apg 4,13.29.31.
} 
Für viele ist ein katholisches Gebetshaus - auch ökumenisch offen ungewohnt neu. Zugleich und weitgehend unabhängig voneinander entstehen aber in diesen Jahrzehnten immer mehr christliche Gebetshäuser, in der Mehrzahl ohne konfessionelle Bindung und fast durchwegs in der Weise von Basisbewegungen: auch wo die Leiter Großkirchen angehören, unabhängig von kirchlicher Hierarchie und finanziert durch Spenden. Verbunden damit gewinnt die alte Vision einer "ewigen Anbetung", die seit vielen Jahrhunderten in Klöstern verbreitet ist und auch in pietistischen Bewegungen eine Geschichte hat, in neuen Formen eine neue Aktualität, und zwar vor allem unter jungen Christen. »24-7«- also 24 Stunden an 7 Tagen in der Woche - wurde zu einer elektrisierenden Parole, die immer mehr christliche Bewegungen erfasst. ${ }^{24}$

Wenn Gebetszeiten nicht nach zwei Stunden enden, sondern über viele Stunden und ganze Nächte durchgehalten werden, ändert sich auch die Form des Betens. In katholischen Bewegungen ist das Gebet vor dem ausgesetzten Allerheiligsten verbreitet, bei freier Gestaltung durch jene Personen, die sich für die jeweiligen Zeiten eingetragen haben. ${ }^{25}$ Eine Tendenz zum charismatischen Beten zeigt sich am Liedgut und Formen eines freien, oft emotionalen Betens - oft in kraftvoller Fürbitte für bestimmte Anliegen, dann wieder Zeiten der Stille und Entspannung. Solches 24-7-Gebet findet nicht nur in Kirchen und Kapellen statt. Vorzugsweise werden Räume mit Gebetsschemeln und gemütlichen Sitzgelegenheiten ausgewählt, an den Wänden Zettel mit Notizen für Gebetsanliegen. Liegen am

\footnotetext{
${ }^{24}$ Vgl. Dave Roberts, Pete Greig, Red Moon Rising. Wenn Freunde anfangen zu träumen und zu beten - Die Geschichte von 24-7 Prayer, Wuppertal ${ }^{5} 2014$ (engl. Original: 2004). Im Internet: http://www.24-7prayer.com. Eine leitende Idee ist dabei: Das Feuer des Gebets soll nicht mehr verlöschen, und zwar nicht nur zur Heiligung der Menschen und der Erneuerung der Gesellschaft, sondern vor allem, um Gott die Ehre zu geben. Die Wege, dieses Ideal zu verwirklichen, sind unterschiedlich. Nach dem Ansatz der 24-7-Bewegung werden Namenslisten ins Internet gelegt, um für bestimmte Tage oder Wochen eine ununterbrochene Präsenz von Betern an bestimmten Orten und durchgängig über viele Orte zu gewährleisten. Andere gründen Gebetshäuser und bemühen sich, so viele feste Mitwirkende zu gewinnen, dass ein beständiges 24-7-Gebet möglich wird. In Deutschland gibt es das Gebetshaus Augsburg, wo seit zwei Jahren Tag und Nacht ununterbrochen Gebet stattfindet (im Internet: http://gebetshaus.org). Dieses Gebetshaus engagiert sich auch für dieVernetzung von Gebetshäusern verschiedener Konfessionen in Europa. Im Frühjahr 2014 organisierte es erstmals ein Treffen mit Vertretern von über 100 Gebetshäusern aus über 30 Nationen.

${ }^{25} \mathrm{Vgl}$. in Österreich die Loretto-Bewegung mit einer charismatischen, eucharistischen, marianischen und ökumenischen Ausrichtung. http://de.wikipedia.org/wiki/ Loretto_Gemeinschaft sowie die Homepage: http://loretto.at
} 
Boden und entspanntes Sitzen - wie Martas Schwester Maria "zu den Füßen Jesu« - entspricht dem Bedürfnis von jungen Menschen, die oft viele Stunden im Gebet verbringen. Die durchgängige Erfahrung ist, dass die Ehrfurcht vor der Präsenz Gottes dabei keineswegs verloren geht.Vielmehr entsteht eine "heilige Atmosphäre«, die Menschen dazu einlädt, zu bleiben.

Dass diese vertiefte Innigkeit offen ist, sich mit sozialer Offenheit zu verbinden, kann eine kleine Bewegung junger katholischer Beter in Salzburg bezeugen. ${ }^{26}$ Durch die Zeit im Gebetsraum im Glauben und in der Liebe gestärkt, folgten sie mitten in der Nacht dem Ruf des Hohelieds: "Aufstehen will ich, die Stadt durchstreifen, die Gassen und Plätze, ihn suchen, den meine Seele liebt " (Hld 3,2). So konnten sie mühelos auf "gestrandete" Menschen - z.B. alkoholisierte Jugendliche - zugehen, in Gespräch und Gebet, und wer wollte, den nahmen sie mit in den Gebetsraum. Mancher traute seinen Augen nicht, als er am nächsten Tag in heiliger Atmosphäre, mit dem Blick auf die Monstranz aus seinem Rausch erwachte. Auch $\mathrm{zu}$ Lebenswenden und Bekehrungen von Menschen ist es auf diese Weise gekommen. ${ }^{27}$

Solche "junge" Formen, für die es auch im protestantischen Bereich Beispiele gibt ${ }^{28}$, stehen quer zu einer verbreiteten europäisch-großkirchlichen Zurückhaltung. Wird hier nicht Menschen ungefragt das Evangelium aufgedrängt? Andererseits entsprechen sie dem Bild einer wesentlich missionarischen und evangelisierenden Kirche, die in der katholischen Kirche seit dem Zweiten Vatikanum von lehramtlichen Texten nochmals stärker betont wird, und näherhin dem Anliegen von Papst Franziskus, Kirche dürfe sich nicht in die Sakristei einsperren, sondern müsse auf die Straße gehen. Hier stoßen wir auf das geistgewirkte Phänomen einer Steigerung von Innen und Außen: Die fromme Innerlichkeit »in der eigenen Kammer" (Mt 6,6) weckt ein Feuer der Liebe - von Gottesliebe zu Nächstenliebe -, das auf die Straße hinausdrängt und die natürlichen Barrieren zu Menschen jenseits des eigenen Milieus überwindet. Die größere Tiefe ist die größere Weite. Gewiss sind auch

\footnotetext{
${ }^{26}$ Es handelt sich um eine Gruppe innerhalb der Loretto-Bewegung, die sich in jugendlichem Übermut "AFFEn« nennt, als Akronym für "Adoration Fighters for Eternity«.

${ }^{27}$ Vgl. das Zeugnis von »Dave« als Video im Internet: http://www.loretto.at/pfingsten/video/pfingsten-13-zeugnis-dave

${ }^{28}$ Vgl. Zeugnisse von Straßenevangelisierung ausgehend von temporären Gebetsräumen unter anderem auf der "Party-Insel" Ibiza: http://www.24-7prayer.com/ community/ibiza sowie die Video-Dokumentation: https://www.youtube.com/ watch? $\mathrm{v}=$ TvlKfiMRolY
} 
beim genannten Beispiel der jungen Salzburger Christen kritische Rückfragen angebracht, für die allerdings eine Unterscheidung aus der Mitte einer charismatisch orientierten Spiritualität möglich ist: Zieht es mich auf die Straße, weil ich etwas Verrücktes erproben will, oder zieht mich gemäß dem Hohelied eine überströmende Liebe, die ein Charisma - Gabe und Aufgabe zugleich - ist und die Barrieren zu ganz anderen Menschen überwindet, sodass sie selber (nicht die eigenen Absichten, und auch nicht der Zustand der Anderen) im Mittelpunkt stehen?

Auch in der Form der Musik gibt es Änderungen. Wer viele Stunden in der Woche musikalisch anbetet, stößt auch mit dicken Liederbüchern bald an Grenzen. Wichtige Anregungen kommen hier vom freikirchlichen International House of Prayer (IHOP) aus Kansas City, wo seit vielen Jahren ein 24-Stunden-Gebet unter der Leitung von mittlerweile über hundert MitarbeiterInnen praktiziert wird. ${ }^{29}$ Hier entstand eine Form eines improvisierten gemeinschaftlich-musizierenden Wechselgesangs ausgehend von zuvor gelesenen biblischen Versen. Unter dem Namen Harp \& Bowl - in Anlehnung an Offb 5,8: Harfen und Schalen mit Gebet - findet diese musikalische Form zunehmend Verbreitung unter jungen charismatischen Christen in Europa. Auf diese Weise kann in spontan entstehenden Melodiefolgen ein biblischer Vers über zwanzig Minuten meditativ durchbetet werden, bis dann ein neues Gebetsanliegen mit neuem Grundmotiv folgt.

Mit guten Musikern kann damit in Gebetshäusern ein eindrucksvoller, ununterbrochener Klangteppich entstehen, verbunden mit einer starken Präsenz von Gotteswort und Glaubensbekundung. Für Menschen, die viele Stunden in der Woche aktiv oder als Gäste dabei sind, ist hier mit starken, auch suggestiven Wirkungen zu rechnen, die sehr unterschiedlich sein können und wohl noch genauer untersucht werden müssen. Ausgedehnte Zeiten der Stille - für ein Hören auf Gott und ein Wahrnehmen seiner selbst in der Gegenwart Gottes - scheinen mir für eine christliche Gebetshauskultur jedenfalls unverzichtbar zu sein.

_ Prof. Dr. Willibald Sandler ist ao. Professor für Dogmatik am Institut für Systematische Theologie an der Universität Innsbruck.

\footnotetext{
${ }^{29}$ Vgl. im Internet: http://en.wikipedia.org/wiki/International_House_of_Prayer, sowie die Homepage http://www.ihopkc.org
} 